\title{
Entropic representation in the theory of molecular electronic structure
}

\author{
Roman F. Nalewajski
}

Received: 6 July 2012 / Accepted: 6 September 2012 / Published online: 27 September 2012 (C) The Author(s) 2012. This article is published with open access at Springerlink.com

\begin{abstract}
The entropic perspective on the molecular electronic structure is investigated. Information-theoretic description of electron probabilities is extended to cover the complex amplitudes (wave functions) of quantum mechanics. This analysis emphasizes the entropic concepts due to the phase part of electronic states, which generates the probability current density, thus allowing one to distinguish the information content of states generating the same electron density and differing in their current densities. The classical information measures of Fisher and Shannon, due to the probability/density distributions themselves, are supplemented by the nonclassical terms generated by the wave-function phase or the associated probability current. A complementary character of the Fisher and Shannon information measures is explored and the relationship between these classical information densities is derived. It is postulated to characterize also their nonclassical (phase/current-dependent) contributions. The continuity equations of the generalized information densities are examined and the associated nonclassical information sources are identified. The variational rules involving the quantum-generalized Shannon entropy, which generate the stationary and timedependent Schrödinger equations from the relevant maximum entropy principles, are discussed and their implications for the system "thermodynamic" equilibrium states are examined. It is demonstrated that the lowest, stationary "thermodynamic" state differs from the true ground state of the system, by exhibiting the space-dependent phase, linked to the modulus part of the wave function, and hence also a nonvanishing probability current.
\end{abstract}

\footnotetext{
R. F. Nalewajski (区)

Department of Theoretical Chemistry,

Jagiellonian University, R. Ingardena 3,

30-060 Cracow, Poland

e-mail: nalewajs@chemia.uj.edu.pl
} 
Keywords Electronic structure theory - Information continuity equations · Maximum entropy principle · Nonclassical entropy · Quantum Fisher information · Quantum mechanics · Schrödinger equation • "Thermodynamic" states

\section{Introduction}

In the quantum mechanical description of, say, a single (spinless) particle the probability density, which defines the classical (Fisher [1] or Shannon [2]) information content of the particle spatial distribution, is determined solely by the moduluspart of the complex wave function. The phase-part of the latter, which generates the probability current, was shown to give rise to additional, nonclassical term in the gradient (Fisher) measure of the information density of complex quantum states, proportional to the square of the probability current [3]. This generalized measure allows one to distinguish the amount of information in two states exhibiting the same particle density and differing in their phases (currents). A similar extension of the classical Shannon concept has been suggested [4]. This phase aspect of the molecular quantum mechanics reflects its "entropic" aspect, which still remains largely unexplored.

In examining this information side of the ground state electronic distribution in molecules one ultimately arrives at more basic questions about a possible existence of general information principles governing this equilibrium electron distribution in molecular systems. It has already been argued elsewhere [3,5] that the stationary Schrödinger equation (SE), the eigenvalue problem of the electronic Hamiltonian in the Born-Oppenheimer (adiabatic) approximation, results from the constrained information principle of the generalized Fisher information, formulated in terms of the complex probability amplitude of quantum mechanics (wave function). This Extreme Physical Information (EPI) principle [5] contains both the "physical" (interaction) and "geometrical" (normalization) constraints. It is also intriguing to find whether the molecular systems tend to maximize in their ground states the separate ionic (deterministic) and covalent (indeterministic) bond components [6] within the Orbital Communication Theory, e.g., [7-9] and refs. therein.

It is the main goal of the present work to examine general implications of such a generalized IT description of molecular states. In particular, the relevant information currents and sources will be identified in the context of the associated probability and phase continuity equations. The physically constrained information principles will be used to reconstruct the Schrödinger equations (stationary and time-dependent) of quantum mechanics. In present work we focus on EPI rule using the nonclassical Shannon entropy [4]. The lowest entropy-equilibrium state exhibiting the ground-state probability density will be examined. This "thermodynamic" state will be shown to exhibit the space-dependent phase linked to the stationary probability density, and hence the nonvanishing currents, thus differing from the lowest eigenstate of the system Hamiltonian. 


\section{Classical information measures, phase current and quantum information continuity}

Let us briefly summarize the classical information/entropy quantities, related to probability distributions. We begin with the gradient measure of Fisher [1,3,5], defined for the local events of finding an electron at $\boldsymbol{r}$. In the probability distribution $p(\boldsymbol{r})$, the shape factor of the electron density $\rho(\boldsymbol{r})=N p(\boldsymbol{r})$, this approach introduces the classical Fisher information, also called the intrinsic accuracy, reminiscent of the familiar von Weizsäcker [10] inhomogeneity correction to the kinetic energy functional in the Thomas-Fermi-Dirac theory:

$$
I[p]=\int[\nabla p(\boldsymbol{r})]^{2} / p(\boldsymbol{r}) d \boldsymbol{r} \equiv \int f^{\text {clas. }}(\boldsymbol{r}) d \boldsymbol{r} .
$$

It characterizes the effective "localization" (compactness) of the random (position) variable around its average value. For example, in the normal (Gaussian) distribution the Fisher information measures the inverse of its variance, i.e., the invariance. The functional $I[p]$ can be simplified, when expressed in terms of its classical aplitude $A(\boldsymbol{r})=\sqrt{p(\boldsymbol{r})}$ :

$$
I[p]=4 \int[\nabla A(\boldsymbol{r})]^{2} d \boldsymbol{r} \equiv I[A] .
$$

In order to cover the quantum (complex) amplitudes (wave functions) this classical measure has to be appropriately generalized by using the square of the modulus of the wave-function gradient [3]. For example, for a single particle of mass $m$, when $p(\boldsymbol{r})=\psi^{*}(\boldsymbol{r}) \psi(\boldsymbol{r})=|\psi(\boldsymbol{r})|^{2}$,

$$
I[\psi]=4 \int|\nabla \psi(\boldsymbol{r})|^{2} d \boldsymbol{r}=8\left(m / \hbar^{2}\right) T[\psi] \equiv \int f(\boldsymbol{r}) d \boldsymbol{r},
$$

where $T[\psi]$ stands for the expectation value of the particle kinetic energy in state $\psi$. Therefore, this local measure of Fisher, proportional to the particle average kinetic energy, probes the length of the amplitude gradient $\nabla \psi(\boldsymbol{r})$.

The other popular information measure has been introduced by Shannon [2]. This complementary descriptor of the average information content in the continuous probability distribution $p(\boldsymbol{r})$, or in its discrete resolution $p(\boldsymbol{r})=\sum_{i} p_{i}(\boldsymbol{r})$ of the orbital events in the molecule generating the condensed probability vector

$$
\begin{gathered}
\boldsymbol{p}=\left\{p_{i}=\int p_{i}(\boldsymbol{r}) d \boldsymbol{r}\right\}, \quad \sum_{i} p_{i}=\int p(\boldsymbol{r}) d \boldsymbol{r}=1, \\
S[p]=-\int p(\boldsymbol{r}) \log p(\boldsymbol{r}) d \boldsymbol{r} \equiv \int s^{\text {clas. }}(\boldsymbol{r}) d \boldsymbol{r} \text { or } \quad S(\boldsymbol{p})=-\sum_{i} p_{i} \log p_{i},
\end{gathered}
$$

called the Shannon entropy, reflects the indeterminacy ("spread") of the random variable involved around its average value. It measures the average amount of the 
information received, when this uncertainty is removed be an appropriate "localization" experiment.

To summarize, the classical Fisher information for locality events reflects the effective narrowness ("order") of the probability distribution $p(\boldsymbol{r})$, while the classical Shannon entropy monotonically increases with the spread ("disorder") of the Gaussian function, thus providing a measure of its uncertainty/indeterminacy content. The intrinsic accuracy and Shannon entropy thus describe the complementary facets of the probability distribution.

These classical measures probe the real probability amplitude $A(\boldsymbol{r})$, while the appropriate quantum extensions, e.g., that defined in Eq. (3), introduce an additional dependence upon the probability current $[3,6]$ related to the gradient of the phase factor of molecular states. For simplicity, we consider a single particle moving in an external potential $v(\boldsymbol{r})$, described by the Hamiltonian

$$
\mathrm{H}(\boldsymbol{r})=-\left(\hbar^{2} / 2 m\right) \nabla^{2}+v(\boldsymbol{r}),
$$

e.g., an electron in the Born-Oppenheimer (adiabatic) description, experiencing Coulomb forces due to the atomic nuclei in their fixed positions. The particle is in the quantum state described by the complex wave function

$$
\psi(\boldsymbol{r})=R(\boldsymbol{r}) \exp [i \Phi(\boldsymbol{r})]=R(\boldsymbol{r})[\cos \Phi(\boldsymbol{r})+i \sin \Phi(\boldsymbol{r})]=\operatorname{Re}[\psi(\boldsymbol{r})]+i \operatorname{Im}[\psi(\boldsymbol{r})],
$$

in which the (real) modulus $R(\boldsymbol{r})$ and phase $\Phi(\boldsymbol{r})$ functions determine the state two fundamental "degrees-of-freedom".

In the familiar Born interpretation of quantum mechanics they determine the particle spatial probability density, $p(\boldsymbol{r})=\psi(\boldsymbol{r}) \psi^{*}(\boldsymbol{r})=R^{2}(\boldsymbol{r})$, with $R$ thus representing the classical amplitude of this probability distribution, and the current density

$$
\boldsymbol{j}(\boldsymbol{r}) \equiv p(\boldsymbol{r}) \boldsymbol{V}(\boldsymbol{r})=(\hbar / m) p(\boldsymbol{r}) \nabla \Phi(\boldsymbol{r})
$$

Therefore, the current density per particle $(\boldsymbol{j} / p) \equiv \boldsymbol{V}$, measuring the local speed $\boldsymbol{V}$ of this probability fluid, is completely determined by the gradient of the phase part of the system wave function,

$$
\begin{aligned}
\Phi(\boldsymbol{r}) & =(2 i)^{-1} \ln \left[\psi(\boldsymbol{r}) / \psi^{*}(\boldsymbol{r})\right], \\
\nabla \Phi(\boldsymbol{r}) & =p(\boldsymbol{r})^{-1}\left\{(2 i)^{-1}\left[\psi^{*}(\boldsymbol{r}) \nabla \psi(\boldsymbol{r})-\psi(\boldsymbol{r}) \nabla \psi^{*}(\boldsymbol{r})\right]\right\} \\
& =p(\boldsymbol{r})^{1} \operatorname{Im}\left[\psi^{*}(\boldsymbol{r}) \nabla \psi(\boldsymbol{r})\right]=(m / \hbar) \boldsymbol{V}(\boldsymbol{r}) .
\end{aligned}
$$

The probability current thus explores the weighted gradient of the wave function phase, related to the imaginary part (Im) of the product $\psi^{*} \nabla \psi$. It also follows from the preceding equation that $\nabla \Phi$ measures the $(m / \hbar)$-scaled current velocity $\boldsymbol{V}$.

The generalized Fisher measure [3] of the information content [Eq. (3)] contains both the classical contribution $I[p]$ [Eq. (1)], due to the probability density $p(r)$, and 
the nonclassical (quantum) correction $I[\boldsymbol{j}]$ involving the current density $\boldsymbol{j}(\boldsymbol{r})$ :

$$
\begin{aligned}
I[\psi] & =4 \int|\nabla \psi(\boldsymbol{r})|^{2} d \boldsymbol{r}=I[p]+4 \int p(\boldsymbol{r})[\nabla \Phi(\boldsymbol{r})]^{2} d \boldsymbol{r} \\
& =I[p]+4(m / \hbar)^{2} \int \boldsymbol{j}^{2}(\boldsymbol{r}) / p(\boldsymbol{r}) d \boldsymbol{r} \equiv I[p]+I[\boldsymbol{j}] \equiv I[p, \boldsymbol{j}] .
\end{aligned}
$$

In general, this quantum gradient measure, related to the system kinetic energy, is not conserved, and exhibits the following full time dependence [9]:

$$
\begin{aligned}
d I[p, \boldsymbol{j}] / d t & =\int \frac{\partial I[p, \boldsymbol{j}]}{\partial p(\boldsymbol{r})} \frac{d p(\boldsymbol{r})}{d t} d \boldsymbol{r}+\int \frac{\partial I[p, \boldsymbol{j}]}{\partial \boldsymbol{j}(\boldsymbol{r})} \cdot \frac{d \boldsymbol{j}(\boldsymbol{r})}{d t} d \boldsymbol{r} \\
& =\int \frac{\partial I[\boldsymbol{j}]}{\partial \boldsymbol{j}(\boldsymbol{r})} \cdot \frac{d \boldsymbol{j}(\boldsymbol{r})}{d t} d \boldsymbol{r}=\frac{8 m}{\hbar^{2}} \int \boldsymbol{j}(\boldsymbol{r}) \cdot \boldsymbol{F}(\boldsymbol{r}) d \boldsymbol{r} \equiv \int \frac{d f(\boldsymbol{r})}{d t} d \boldsymbol{r},
\end{aligned}
$$

where the force acting on the particle $\boldsymbol{F}(\boldsymbol{r})=-\nabla v(\boldsymbol{r})$. In the preceding equation we have used the familiar probability-continuity equation:

$$
d p(\boldsymbol{r}) / d t \equiv \sigma_{p}(\boldsymbol{r})=\partial p(\boldsymbol{r}) / \partial t+\nabla \cdot \boldsymbol{j}(\boldsymbol{r})=0 \quad \text { or } \quad \partial p(\boldsymbol{r}) / \partial t=-\nabla \cdot \boldsymbol{j}(\boldsymbol{r}) .
$$

It expresses the local balance in probability redistributions and signifies the sourceless $\left(\sigma_{p}(\boldsymbol{r})=0\right)$ probability redistributions in molecular systems: the local change in the probability density is solely due to the probability outflow measured by the negative divergence of the probability current density. It also follows from the preceding equation that the overall probability normalization, i.e., the squared norm of the wave function, is conserved in time:

$$
d / d t\left[\int p(\boldsymbol{r}) d \boldsymbol{r}\right]=0 .
$$

The source $\sigma_{f}$ of this quantum-generalized Fisher information thus reads:

$$
\frac{d f(\boldsymbol{r})}{d t}=\sigma_{f}=\frac{8 m}{\hbar^{2}} \boldsymbol{j}(\boldsymbol{r}) \cdot \boldsymbol{F}(\boldsymbol{r}) .
$$

Hence, the local production of this quantum measure of the Fisher information is proportional to the product of the local force and flow vectors. This is in perfect analogy to the familiar expression for the entropy source in irreversible thermodynamics [11], given by the sum of products of the corresponding rates of change (or flows) of extensive quantities (thermodynamic fluxes) and the conjugate gradients or differences of intensive "forces" (thermodynamic affinities). In the nondegenerate ground state, for which the time dependent phase is position independent, the current and its information contribution identically vanish, so that the current correction $I[j]$ to the gradient (Fisher) measure of information should be important in the degenerate stationary states of the particle and in its general (nonstationary) quantum states. 
One similarly introduces [4] the related phase-flow concept, associated with the other degree-of-freedom of quantum states. By symmetry, it can be expected to be related to the gradient of the modulus part $R(\boldsymbol{r})=[p(\boldsymbol{r})]^{-1 / 2}$ of the wave function,

$\nabla R(\boldsymbol{r})=p(\boldsymbol{r})^{-1 / 2}\left\{\left[\psi^{*}(\boldsymbol{r}) \nabla \psi(\boldsymbol{r})+\psi(\boldsymbol{r}) \nabla \psi^{*}(\boldsymbol{r})\right] / 2\right\}=p(\boldsymbol{r})^{-1 / 2} \operatorname{Re}\left[\psi^{*}(\boldsymbol{r}) \nabla \psi(\boldsymbol{r})\right]$,

measured by the real part (Re) of $\psi^{*} \nabla \psi$, or the gradients of both factors in $\psi(\boldsymbol{r})$.

We recall that the current concept, a crucial component of the probability continuity equation in quantum mechanics, emerges in the context of the time dependence of the system wave function, determined by the Schrödinger equation or its complex conjugate:

$$
\mathrm{H}(\boldsymbol{r}) \psi(\boldsymbol{r})=i \hbar \partial \psi(\boldsymbol{r}) / \partial t \text { and } \mathrm{H}(\boldsymbol{r}) \psi^{*}(\boldsymbol{r})=-i \hbar \partial \psi^{*}(\boldsymbol{r}) / \partial t
$$

Via their straightforward manipulations one obtains from their "weighted" difference the familiar equation for the local probability continuity,

$$
\begin{aligned}
\partial p(\boldsymbol{r}) / \partial t & =\psi^{*}(\boldsymbol{r})[\partial \psi(\boldsymbol{r}) / \partial t]+\psi(\boldsymbol{r})\left[\partial \psi^{*}(\boldsymbol{r}) / \partial t\right]=2 R(\boldsymbol{r})[\partial R(\boldsymbol{r}) / \partial t] \\
& =-\nabla \cdot \boldsymbol{j}(\boldsymbol{r})=-(\hbar / m)\left[2 R(\boldsymbol{r}) \nabla R(\boldsymbol{r}) \cdot \nabla \Phi(\boldsymbol{r})+R^{2}(\boldsymbol{r}) \Delta \Phi(\boldsymbol{r})\right] .
\end{aligned}
$$

Thus, the local change in the probability density (1.h.s. of the preceding equation) is solely due to the probability outflow (r.h.s.), the magnitude of which is measured by the divergence of the probability-current density. It also follows from this probability-continuity equation that the local time-derivative of the modulus factor $R$ reads:

$$
\begin{aligned}
\partial R(\boldsymbol{r}) / \partial t & =[2 R(\boldsymbol{r})]^{1} \partial p(\boldsymbol{r}) / \partial t=-[2 R(\boldsymbol{r})]^{1} \nabla \cdot \boldsymbol{j}(\boldsymbol{r}) \\
& =-(\hbar / m)\{\nabla R(\boldsymbol{r}) \cdot \nabla \Phi(\boldsymbol{r})+[R(\boldsymbol{r}) / 2] \Delta \Phi(\boldsymbol{r})\} .
\end{aligned}
$$

The "weighted" sum of the Schrödinger equations (16) similarly gives:

$$
\begin{aligned}
\psi^{*}(\boldsymbol{r})[\partial \psi(\boldsymbol{r}) / \partial t]-\psi(\boldsymbol{r})\left[\partial \psi^{*}(\boldsymbol{r}) / \partial t\right] & =2 i p(\boldsymbol{r})[\partial \Phi(\boldsymbol{r}) / \partial t] \\
& =(i \hbar / m)\left\{R(\boldsymbol{r}) \Delta R(\boldsymbol{r})-[R(\boldsymbol{r}) \nabla \Phi(\boldsymbol{r})]^{2}\right\}
\end{aligned}
$$

It generates the time-derivative of the wave-function phase,

$$
\partial \Phi(\boldsymbol{r}) / \partial t=(\hbar / 2 m)\left\{R^{-1}(\boldsymbol{r}) \Delta R(\boldsymbol{r})-[\nabla \Phi(\boldsymbol{r})]^{2}\right\}-v(\boldsymbol{r}) / \hbar
$$

including in the last term the external potential contribution. It thus follows from Eqs. (18) and (20) that the Schrödinger equation gives rise to a coupled dynamics of both these components of the complex wave function. Notice, however, that only the phase function explicitly depends on the system external potential. It is our goal now to express this phase derivative as a combination of the corresponding divergence of the 
related phase-current and the accompanying phase-source terms, in the associated phase-continuity equation.

It should be recalled that the continuity balance expresses some basic conservation law. One could associate the following expectation (average) value of the system phase corresponding to the particle probability distribution $p(\boldsymbol{r})$ :

$$
\langle\Phi\rangle=\int p(\boldsymbol{r}) \Phi(\boldsymbol{r}) d \boldsymbol{r} \equiv \int \varphi(\boldsymbol{r}) d \boldsymbol{r},
$$

with $\varphi(\boldsymbol{r})$ standing for the particle phase density at $\boldsymbol{r}$, and the phase factor $\Phi(\boldsymbol{r})$ now representing the associated density per electron. In a search for the source $\sigma_{\varphi}(\boldsymbol{r})$ of the phase distribution $\varphi(\boldsymbol{r})$ one again calculates the total time derivative (for the moving monitoring space element),

$$
d \varphi(\boldsymbol{r}) / d t \equiv \sigma_{\varphi}(\boldsymbol{r})=\partial \varphi(\boldsymbol{r}) / \partial t+\nabla \cdot \boldsymbol{J}_{\varphi}(\boldsymbol{r})
$$

with the partial derivative measuring the local change in the fixed monitoring volume around $\boldsymbol{r}$ and the divergence term representing the phase inflow due to the associated current $\boldsymbol{J}_{\varphi}$. Since $\varphi(\boldsymbol{r})$ depends upon both the probability density $p(\boldsymbol{r})$ and the local phase $\Phi(\boldsymbol{r})$,

$$
d \varphi / d t=\Phi(d p / d t)+p(d \Phi / d t)=p(d \Phi / d t)
$$

since the source $\sigma_{p}$ vanishes [Eq. (12)]. Therefore, the continuity equation (22) is fully determined by the balance equation for the phase factor itself:

$$
d \Phi / d t \equiv \sigma_{\Phi}=\partial \Phi / \partial t+\nabla \cdot \boldsymbol{J}_{\Phi}
$$

Identification of the phase-current $\boldsymbol{J}_{\Phi}$ and the associated source $\sigma_{\Phi}$, both conforming to the time derivative of Eq. (20) derived from the Schrödinger equation (16), is not unique and an adoption of the specific form of the phase current implies the conjugate form of the source. Since the speed $(\boldsymbol{j} / p)=\boldsymbol{V}$ of the probability fluid reflects the gradient of the complementary phase factor $\Phi$, one would expect the phase current to involve the gradient of the amplitude $R$, or the gradients of both these degrees-offreedom of the quantum state $\psi$.

One observes the following derivative identities containing terms in r.h.s. of Eq. (20):

$$
\begin{aligned}
& \nabla \cdot(\Phi \nabla \Phi)=(\nabla \Phi)^{2}+\Phi \Delta \Phi \text { and } \\
& \nabla \cdot(\nabla \ln R)=\nabla \cdot\left(R^{-1} \nabla R\right)=R^{-1} \Delta R-\left(R^{-1} \nabla R\right)^{2} .
\end{aligned}
$$

Therefore, introducing the phase-current $\boldsymbol{J}_{\Phi}$ involving the gradients of both wave function components,

$$
J_{\Phi}=-(\hbar / 2 m)\left(R^{-1} \nabla R-\Phi \nabla \Phi\right)
$$


gives the following source of Eq. (24):

$$
\sigma_{\Phi}=(\hbar / 2 m)\left[(\nabla \ln R)^{2}+\Phi \Delta \Phi\right]-v / \hbar
$$

Similarly, by linking the current solely to the gradient of the amplitude factor,

$$
\boldsymbol{J}_{\Phi}^{\prime}=-(\hbar / 2 m)\left(R^{-1} \nabla R\right)=-(\hbar / 2 m) \nabla \ln R
$$

yields

$$
\sigma_{\Phi}^{\prime}=(\hbar / 2 m)\left[(\nabla \ln R)^{2}-(\nabla \Phi)^{2}\right]-v / \hbar
$$

In the stationary, ground state of one-particle system,

$$
\Psi_{0}(\boldsymbol{r}, t)=R_{0}(\boldsymbol{r}) \exp \left[i \Phi_{0}(\boldsymbol{r}, t)\right]=R_{0}(\boldsymbol{r}) \exp \left(-i E_{0} t / \hbar\right) \equiv R_{0}(\boldsymbol{r}) \exp \left(-i \omega_{0} t\right),
$$

corresponding to the sharply specified energy $E=E_{0}$, the local phase is equalized throughout the whole physical space, $\Phi_{0}(\boldsymbol{r}, t)=\Phi_{0}(t)=-\omega_{0} t$, and hence: $\nabla \Phi_{0}=0$ and $\langle\Phi\rangle=\Phi_{0}(\boldsymbol{t})$. In such states both definitions of the phase current and the associated source become identical and time independent:

$$
\begin{aligned}
& \boldsymbol{J}_{\Phi}\left[\psi_{0}\right]=\boldsymbol{J}_{\Phi}^{\prime}\left[\psi_{0}\right]=-(\hbar / 2 m) R_{0}^{-1} \nabla R_{0}=-(\hbar / 2 m) \nabla\left(\ln R_{0}\right), \\
& \sigma_{\Phi}\left[\psi_{0}\right]=\sigma_{\Phi}^{\prime}\left[\psi_{0}\right]=(\hbar / 2 m)\left(\nabla \ln R_{0}\right)^{2}-v / \hbar
\end{aligned}
$$

This phase equalization in the stationary quantum state is related to the associated equalization of the system local energy,

$$
\varepsilon(\boldsymbol{r})=\psi(\boldsymbol{r})^{-1} \mathrm{H}(\boldsymbol{r}) \psi(\boldsymbol{r}),
$$

at the ground-state level:

$$
\varepsilon_{0}(\boldsymbol{r})=\psi_{0}^{-1}(\boldsymbol{r}) \mathrm{H}(\boldsymbol{r}) \psi_{0}(\boldsymbol{r})=E_{0} .
$$

\section{Quantum extension of Shannon entropy}

It follows from the preceding section that a similar generalization of the classical Shannon entropy $S[p]$ is required in quantum mechanics, in order to include relevant current contribution [4]. Let us briefly summarize the basis of such a quantum extension of this complementary information measure.

It follows from Eq. (10) that both the electron distribution $p(\boldsymbol{r})$ and its current $\boldsymbol{j}(\boldsymbol{r})$ determine the resultant quantum Fisher information content $I[p, j]$. Its first, classical part $I[p]$ explores the information contained in the probability distribution, while the nonclassical part $I[j]$ measures the gradient information in the probability current, 
i.e., the phase gradient of Eq. (9). Thus, the quantum Fisher functional $I[\psi]$ symmetrically probes the gradient content of both aspects of the complex wave-function:

$$
I[p]=\int\left(\frac{\nabla p}{\sqrt{p}}\right)^{2} d \boldsymbol{r} \equiv \int(\bar{\nabla} p)^{2} d \boldsymbol{r}, \quad I[\boldsymbol{j}]=\int\left(\frac{2 \mu \boldsymbol{j}}{\hbar \sqrt{p}}\right)^{2} d \boldsymbol{r} \equiv \int \overline{\boldsymbol{j}}^{2} d \boldsymbol{r}
$$

The classical Fisher information measures the length of the "reduced" gradient $\bar{\nabla} p$ of the probability density, while the other contribution represents the corresponding "length" of the reduced vector of the probability current density $\overline{\boldsymbol{j}}$. One also observes that this generalized measure becomes identical with the classical functional $I[p]$ only for the stationary quantum states, characterized by the time independent probability amplitude $R(\boldsymbol{r})=\varphi(\boldsymbol{r})$ and the position-independent phase $\Phi(t)=-\omega t$ :

$$
\Psi(\boldsymbol{r}, t)=\varphi(\boldsymbol{r}) \exp (-i E t / \hbar) \equiv \varphi(\boldsymbol{r}) \exp (-i \omega t) .
$$

The two information contributions in Eq. (10) can be alternatively expressed in terms of the real and imaginary parts of the gradient of the wave-function logarithm, $\nabla \ln \psi=(\nabla \psi) / \psi$,

$$
I[p]=4 \int p[\operatorname{Re}(\nabla \ln \psi)]^{2} d \boldsymbol{r} \text { and } I[\boldsymbol{j}]=4 \int p[\operatorname{Im}(\nabla \ln \psi)]^{2} d \boldsymbol{r} .
$$

Thus, these complementary components of the quantum Fisher information have a common interpretation in quantum mechanics, as the $p$-weighted averages of the gradient content of the real and imaginary parts of the logarithmic gradient of the system wave-function. As such they indeed represent a natural quantum (complex) generalization of the classical (real) information concept of Eq. (1).

Let us examine some properties of the density of this generalized Fisher information,

$$
f=\frac{(\nabla p)^{2}}{p}+\frac{4 m^{2}}{\hbar^{2}} \frac{j^{2}}{p}=f^{\text {clas. }}+f^{\text {nclas. }}
$$

or the corresponding density per electron:

$$
\begin{aligned}
\tilde{f} \equiv \frac{f}{p} & =\left(\frac{\nabla p}{p}\right)^{2}+\left(\frac{2 m j}{\hbar p}\right)^{2} \equiv(\tilde{\nabla} p)^{2}+(\tilde{j})^{2} \\
& =\tilde{f}^{\text {clas. }}+\tilde{f}^{\text {nclas. }}=4|(\nabla \psi) / \psi|^{2}=4|\nabla \ln \psi|^{2} \geq 0 .
\end{aligned}
$$

The latter is generated by the squares of the local values of the related quantities per electron, the probability gradient $(\tilde{\nabla} p)^{2}$ and the current density $(\tilde{j})^{2}$, which shape the classical and nonclassical information terms in quantum mechanics. This expression emphasizes the basic equivalence of the roles played by the probability density and its current in shaping the resultant density of the generalized, quantum Fisher information. 
We now examine a possible relation between the classical part of the Fisher information density per electron,

$$
\tilde{f}^{\text {clas. }}=f^{\text {clas. }} / p=[(\nabla p) / p]^{2}=(\nabla \ln p)_{2},
$$

and the associated density perparticle

$$
\tilde{s}^{\text {clas. }}=s^{\text {clas. }} / p=-\ln p,
$$

of the classical Shannon entropy of Eq. (4), $s^{\text {clas. }}=-p \ln p=p \tilde{s}^{\text {clas. }}$. Thus, the logarithm of the probability density shapes the classical part of the Shannon entropy density $\tilde{s}^{\text {clas. }}$, the gradient of which gives $\nabla \tilde{s}^{\text {clas. }}=-(\nabla p) / p$. Hence, these two complementary (classical) information densities per electron are related:

$$
\tilde{f}^{\text {clas. }}=(\nabla \ln p)^{2}=\left(\nabla \tilde{s}^{\text {clas. }}\right)^{2} .
$$

This relation has been used to introduce the unknown nonclassical part $\tilde{s}^{\text {nclas. }}$ of the density per-electron of the generalized Shannon entropy in quantum mechanics [4]:

$$
\tilde{s}[\psi]=\tilde{s}^{\text {clas. }} \cdot[p]+\tilde{s}^{\text {nclas. }} \cdot[p, \Phi] .
$$

Its nonclassical term $\tilde{s}^{\text {nclas. }} \cdot[p, \Phi]=\tilde{s}^{\text {nclas. }}[p, j]$ has been arrived at by postulating that Eq. (41) also holds for the complementary nonclassical contributions:

$$
\begin{aligned}
\tilde{f}^{\text {nclas. }} & =\left(\frac{2 m j}{\hbar p}\right)^{2} \equiv\left(\nabla \tilde{s}^{\text {nclas. }}\right)^{2} \text { or } \\
\nabla \tilde{s}^{\text {nclas. }} & =\frac{2 m j}{\hbar p}=\frac{-i}{p}\left[\psi^{*} \nabla \psi-\psi \nabla \psi^{*}\right]=2 \nabla \Phi .
\end{aligned}
$$

Therefore, the gradient of the nonclassical part of the generalized Shannon entropy is proportional to the probability current per electron, i.e., the velocity of the probability fluid, and hence to an additive constant [see Eq. (8)]

$$
\tilde{s}^{\text {nclas. }}=2 \Phi=-i \ln \left(\psi / \psi^{*}\right) .
$$

We thus conclude that, to physically irrelevant constant, the phase function proper can be regarded as the nonclassical part of the Shannon information density per particle. It then gives a transparent division of the generalized quantum entropy:

$$
\begin{aligned}
& \tilde{s}[\psi]=\tilde{s}^{\text {clas }} \cdot[R]+\tilde{s}^{\text {nclas }} \cdot[\Phi]=-2 \ln R+2 \Phi \text { or } \\
& s[\psi]=s^{\text {clas }} \cdot[p]+s^{n c l a s} \cdot[p, \Phi]=-p \ln p+2 p \Phi=-p \ln p+2 \varphi .
\end{aligned}
$$

The density per particle $\tilde{s}[\psi]$ of the generalized Shannon entropy is thus divided into the familiar classical component $\tilde{s}^{\text {clas. }}[R]$, determined solely by the wave function 
modulus factor $R(\boldsymbol{r})$, and the nonclassical supplement $\tilde{s}^{\text {nclas. }}$ [ $\left.\Phi\right]$, reflecting the actual phase function of the system complex wave function.

Next, let us examine the associated source of such a quantum generalization of the classical Shannon entropy,

$$
\begin{aligned}
S[\psi] \equiv \int s[\psi ; \boldsymbol{r}] d \boldsymbol{r} & =\int s^{\text {clas. }}[p ; \boldsymbol{r}] d \boldsymbol{r}+\int s^{\text {nclas. }}[p, \Phi ; \boldsymbol{r}] d \boldsymbol{r} \\
& =S^{\text {clas. }}[p]+S^{\text {nclas. }}[p, \Phi] \\
& =-\int p(\boldsymbol{r}) \ln p(\boldsymbol{r}) d \boldsymbol{r}+2 \int p(\boldsymbol{r}) \Phi(\boldsymbol{r}) d \boldsymbol{r} \equiv S[p, \Phi] .
\end{aligned}
$$

Again, due to the sourceless character of the probability distribution the classical term generates the vanishing source contribution to this entropy functional and its density:

$$
d S^{\text {clas. }} / d t=\int\left(\frac{\partial S^{\text {clas. }}[p]}{\partial p(\boldsymbol{r})}\right) \frac{d p(\boldsymbol{r})}{d t} d \boldsymbol{r}=0 \text { and } \frac{d s^{\text {clas. }}[p, \boldsymbol{r}]}{d t}=\sigma_{s}^{\text {clas. }}(\boldsymbol{r})=0 .
$$

Hence, for the phase current $\boldsymbol{J}_{\phi}$ of Eq. (26) one obtains

$$
\begin{aligned}
d S[\psi] / d t & =\int \frac{d s[\psi ; \boldsymbol{r}]}{d t} d \boldsymbol{r} \equiv \int \sigma_{s}[\psi ; \boldsymbol{r}] d \boldsymbol{r} \\
& =\int \frac{\partial S^{n c l a s .}[p, \Phi]}{\partial p(\boldsymbol{r})} \frac{d p(\boldsymbol{r})}{d t} d \boldsymbol{r}+\int \frac{\partial S^{\text {nclas. }}[p, \Phi]}{\partial \Phi(\boldsymbol{r})} \frac{d \Phi(\boldsymbol{r})}{d t} d \boldsymbol{r} \\
& =\int \frac{\partial S^{\text {nclas. }}[p, \Phi]}{\partial \Phi(\boldsymbol{r})} \frac{d \Phi(\boldsymbol{r})}{d t} d \boldsymbol{r}=2 \int p(\boldsymbol{r}) \sigma_{\Phi}(\boldsymbol{r}) d \boldsymbol{r}=\int \sigma_{s}^{\text {nclas. }}[\psi ; \boldsymbol{r}] d \boldsymbol{r},
\end{aligned}
$$

where the phase source $\sigma_{\Phi}(\boldsymbol{r})$ is defined in Eq. (27). Alternatively, the current $\boldsymbol{J}_{\Phi}^{\prime}$ and the conjugated source $\sigma_{\Phi}^{\prime}$ can be applied to define this rate of change of the quantum entropy function and its source.

\section{Schrödinger variation rule as information principle}

We begin with a short reminder of the EPI using the Fisher information measure, related directly to the particle kinetic energy, which generates the stationary SE [3]. For the simplest case of a single electron moving in the external potential $v(\boldsymbol{r})$ due to the nuclei in their fixed positions, described by the Coulomb Hamiltonian operator $\mathrm{H}(\boldsymbol{r})=-\left[\hbar^{2} /(2 m)\right] \Delta+v(\boldsymbol{r}) \equiv \mathrm{T}(\boldsymbol{r})+v(\boldsymbol{r})$. Its lowest (nondegenerate) eigenvalue problem, 


$$
\begin{aligned}
\mathrm{H}(\boldsymbol{r}) \psi_{0}(\boldsymbol{r}) & =E_{0} \psi_{0}(\boldsymbol{r}), \\
E_{0} & \left.\left.=\left\langle\psi_{0}|\mathrm{H}| \psi_{0}\right\rangle=\left\langle\psi_{0}|\mathrm{~T}| \psi_{0}\right\rangle+\left\langle\psi_{0}|v| \psi_{0}\right\rangle=T\left[\psi_{0}\right]+V\right] \psi_{0}\right] \equiv T_{0}+V_{0},
\end{aligned}
$$

where, $T_{0}$ and $V_{0}$ denote the corresponding expectation values of the two energy components in the molecular ground-state, then determines the system equilibrium electron/probability density: $\rho_{0}(\boldsymbol{r})=p_{0}(\boldsymbol{r})=\left|\psi_{0}(\boldsymbol{r})\right|^{2}=R_{0}(\boldsymbol{r})^{2}$.

We recall that this SE represents the Euler equation for the optimum ground-state wave function $\psi_{0}$, the distribution amplitude, which results from the familiar variational principle for the minimum electronic energy $E[\psi]=\langle\psi|H| \psi\rangle=T[\psi]$ $+V[\psi]$, combining the average kinetic, $T[\psi]$, and potential, $V[\psi]$, components, respectively, subject to the "geometric" constraint of the wave function (probability) normalization,

$$
\min _{\psi}\left\{\langle\psi|\mathrm{H}| \psi\rangle-E_{0}\langle\psi \mid \psi\rangle\right\} \equiv \min _{\psi} \Omega\left[\psi ; E_{0}\right] \text { or } \delta_{\psi} \Omega\left[\psi ; E_{0}\right]=0,
$$

with the exact electronic energy $E_{0}$ providing the relevant Lagrange multiplier enforcing this subsidiary condition. Indeed, the associated Euler equation directly recovers Eq. (49):

$$
\delta \Omega\left[\psi ; E_{0}\right] /\left.\delta \psi^{*}(\boldsymbol{r})\right|_{\text {opt }}=\left[\mathrm{H}(\boldsymbol{r})-E_{0}\right] \psi_{0}(\boldsymbol{r})=0
$$

It further follows from a straightforward integration by parts that the average kinetic energy is proportional to the generalized Fisher information for locality events, i.e., the quantum intrinsic accuracy [Eq. (3)]:

$$
\begin{aligned}
8 T[\psi] & =\left(\hbar^{2} / m\right)\left\{4 \int|\nabla \psi(\boldsymbol{r})|^{2} d \boldsymbol{r}\right\} \equiv\left(\hbar^{2} / m\right) I[\psi] \text { or } \\
I[\psi] & =\left(8 m / \hbar^{2}\right) T[\psi] .
\end{aligned}
$$

Therefore, the Schrödinger principle of Eq. (50) can in fact be interpreted as the EPI rule for the minimum of the quantum-generalized Fisher information [3,7-9],

$$
\begin{gathered}
\min _{\psi}\left(8 m / \hbar^{2}\right) \Omega\left[\psi ; E_{0}\right]=\min _{\psi}\left\{I[\psi]+\left(8 m / \hbar^{2}\right)\left(V[\psi]-E_{0}\langle\psi \mid \psi\rangle\right)\right\} \\
=\min _{\psi}\left\{I[\psi]+\left(8 m / \hbar^{2}\right)\left(V[\psi]-V_{0}\langle\psi \mid \psi\rangle\right)\right. \\
\left.-\left(8 m / \hbar^{2}\right)\left(E_{0}-V_{0}\right)\langle\psi \mid \psi\rangle\right\} \text { or } \\
\min _{\psi}\left\{I[\psi]+\left(8 m / \hbar^{2}\right)\left(V[\psi]-V_{0}\right)-I_{0}(\langle\psi \mid \psi\rangle-1)\right\},
\end{gathered}
$$

where $I_{0}=\left(8 m / \hbar^{2}\right) T_{0}=I\left[\psi_{0}\right]$ stands for the Fisher information in the system ground-state. 
Thus, the Schrödinger variational principle represents the EPI rule [3,5] in terms of the quantum analog of the classical Fisher information [Eq. (3)]. It contains the "physical" constraint of the fixed potential energy, and the "geometrical" condition of the unit norm of the system optimum wave function. The latter is seen to be enforced by the Lagrange multiplier proportional to the exact ground-state information content $I_{0}$ related to $T_{0}$. It also directly follows from this energy variation principle that for the constrained displacements from the equilibrium state $\psi_{0}$, which conserve $V[\psi]=V_{0}$ and preserve wave-function normalization, $\langle\psi \mid \psi\rangle=1$, the kinetic energy (Fisher information) can only increase, thus confirming the constrained minimum value of the latter in $\psi_{0}$.

Consider now the partial variational problem associated with this information measure, $I[\psi]=I[p, j(\Phi)]$, involving the search for the optimum phase $\Phi$ or the probability current $\boldsymbol{j}(\Phi)=p \nabla \Phi$ [Eq. (7)], for the fixed ground state probability distribution $p=p_{0}$. For the wave function displacements satisfying the physical and geometrical constraints of Eq. (53) this partial variational problem reads:

$$
\begin{aligned}
\min _{\boldsymbol{j}} I\left[p_{0}, \boldsymbol{j}\right] & =\min _{\boldsymbol{j}} \int p^{0}(\boldsymbol{r}) \tilde{f}^{n \text { clas. }}(\boldsymbol{j} ; \boldsymbol{r}) d \boldsymbol{r} \equiv \min _{\boldsymbol{j}}\langle\tilde{f}\rangle_{p_{0}} \\
& =\min _{\Phi} \int p^{0}(\boldsymbol{r})\left[\nabla \tilde{s}^{n c l a s .}(\Phi ; \boldsymbol{r})\right]^{2} d \boldsymbol{r} \equiv \min _{\Phi}\left\langle\left(\nabla \tilde{s}^{n c l a s .}\right)^{2}\right\rangle_{p_{0}} \\
& =\max _{\Phi} S\left[p_{0}, \Phi\right] .
\end{aligned}
$$

In the preceding equation we have used Eq. (43), which recognizes that the nonclassical density per electron of the Fisher information reflects the magnitude of the gradient of the associated Shannon entropy density. Therefore, the minimum of the former, fixing $\boldsymbol{j}=0$ in the system ground state, implies the corresponding minimum (vanishing) length of the gradient of the latter, fixing the space-independent phase

$$
\Phi_{0}(t)=-\left(E_{0} / \hbar\right) t \equiv-\omega_{0} t
$$

thus assuring the maximum smoothness of $\tilde{s}^{\text {nclas. }}$, i.e., the maximum value of $S\left[\psi_{0}\right]=$ $S\left[p_{0}, \Phi_{0}\right]$. This further demonstrates the complementary character of these two nonclassical information measures per electron, for the fixed ground-state probability distribution.

Let us examine this partial, density-constrained maximum entropy principle of Eq. (54) for determining the optimum phase function $\Phi(\boldsymbol{r})$ in some detail. We recall that the nondegenerate ground state of the single particle system is characterized by the vanishing $\Phi_{0}(\boldsymbol{r})=0$, exhibiting only the purely time-dependent phase $\Phi_{0}(t)$ in the full ground-state wave function,

$$
\Psi_{0}(\boldsymbol{r}, t)=R_{0}(\boldsymbol{r}) \exp \left[i \Phi_{0}(t)\right]=R_{0}(\boldsymbol{r}) \exp \left[-i\left(E_{0} / \hbar\right) t\right] \equiv \psi_{0}(\boldsymbol{r}) \exp \left[-i \omega_{0} t\right]
$$


and sharply specified, conserved electronic energy

$$
\begin{aligned}
E_{0} & =E\left[\psi_{0}\right]=\frac{\hbar^{2}}{2 m} \int\left[\nabla R_{0}(\boldsymbol{r})\right]^{2} d \boldsymbol{r}+\int p_{0}(\boldsymbol{r}) v(\boldsymbol{r}) d \boldsymbol{r} \\
& =T\left[\psi_{0}\right]+V\left[\psi_{0}\right]=T_{0}+V_{0} .
\end{aligned}
$$

One also observes that the stationary SE (49) additionally implies the equalization of the local energy:

$$
\varepsilon_{0}(\boldsymbol{r}) \equiv \psi_{0}(\boldsymbol{r})^{-1}\left[\mathrm{H}(\boldsymbol{r}) \psi_{0}(\boldsymbol{r})\right]=E_{0} .
$$

Therefore, either the global energy constraint $E\left[\psi_{0}\right]=E_{0}$ or the local requirement $\varepsilon_{0}(\boldsymbol{r})=E_{0}$ can be used to identify the stationary ground state in quantum mechanics.

One might expect, that admitting the space-dependent phase $\Phi(\boldsymbol{r})$ in the variational wave-function $\psi(\boldsymbol{r})=\mathrm{R}(\boldsymbol{r}) \exp [i \Phi(\boldsymbol{r})]$ of this general maximum-entropy principle, may not conserve the electronic energy in trial variations of the system wave function $\psi\left(\boldsymbol{r} ; p_{0}\right)$. The expression for the expectation value of the Hamiltonian indeed gives:

$$
\begin{aligned}
E[\psi] & =\int R(\boldsymbol{r})^{2} v(\boldsymbol{r}) d \boldsymbol{r}+\frac{\hbar^{2}}{2 m} \\
\times & \left(\int R(\boldsymbol{r})^{2}[\nabla \Phi(\boldsymbol{r})]^{2} d \boldsymbol{r}-(1+i) \int\left\{[\nabla R(\boldsymbol{r})]^{2}+R(\boldsymbol{r}) \nabla R(\boldsymbol{r}) \cdot \nabla \Phi(\boldsymbol{r})\right\} d \boldsymbol{r}\right) .
\end{aligned}
$$

Notice, that the kinetic energy term of this energy expression differs from that in Eq. (57). The physical constraint $E\left[\psi\left(\boldsymbol{r} ; p_{0}\right)\right]=E_{0}$ is also required in this maximum entropy rule to identify the Hamiltonian, i.e., the system itself, in such a thermodynamic-like variational procedure.

The Euler equation resulting from the stationary property of the corresponding auxiliary entropy function,

$$
\delta\left\{S\left[p_{0}, \Phi\right]-\lambda\left(E\left[\psi\left(\boldsymbol{r} ; p_{0}\right)\right]-E_{0}\right)\right\}=0,
$$

with respect to the probability-constrained wave function

$$
\psi\left(\boldsymbol{r} ; p_{0}\right)=R_{0}(\boldsymbol{r}) \exp [i \Phi(\boldsymbol{r})]
$$

or its complex conjugate $\psi^{*}\left(\boldsymbol{r} ; p_{0}\right)=R_{0}(\boldsymbol{r}) \exp [-i \Phi(\boldsymbol{r})]$, which by construction conserve the given ground-state probability distribution

$$
p\left(\boldsymbol{r} ; p_{0}\right)=\psi^{*}\left(\boldsymbol{r} ; p_{0}\right) \psi\left(\boldsymbol{r} ; p_{0}\right) \equiv p_{0}(\boldsymbol{r})=R_{0}(\boldsymbol{r})^{2},
$$


reads:

$$
\begin{aligned}
\frac{\delta S\left[p_{0}, \Phi\right]}{\delta \psi^{*}\left(\boldsymbol{r} ; p_{0}\right)}-\lambda \mathrm{H}(\boldsymbol{r}) \psi\left(\boldsymbol{r} ; p_{0}\right) & =\frac{\partial S^{\text {nclas. }}[p, \Phi]}{\partial \Phi(\boldsymbol{r})} \frac{\partial \Phi(\boldsymbol{r})}{\partial \psi^{*}(\boldsymbol{r})}-\lambda \mathrm{H}(\boldsymbol{r}) \psi\left(\boldsymbol{r} ; p_{0}\right) \\
& =[i-\lambda \mathrm{H}(\boldsymbol{r})] \psi\left(\boldsymbol{r} ; p_{0}\right)=0 .
\end{aligned}
$$

By dividing both sides by $\lambda \neq 0$ one arrives at equation $[(i / \lambda)-\mathrm{H}(\boldsymbol{r})] \psi\left(\boldsymbol{r} ; p_{0}\right)=0$. It is seen to reconstruct the stationary SE for $i / \lambda=E_{0}$, or $\lambda=i / E_{0}$, which then further implies $\Phi(\boldsymbol{r})=0$ in the system true ground state $\psi\left(\boldsymbol{r} ; p_{0}\right)=R_{0}(\boldsymbol{r})=\psi_{0}(\boldsymbol{r})$, i.e., the lowest eigenstate of the Hamiltonian.

Since the average energy is no longer conserved in a general (nonstationary) quantum state $\Psi(\boldsymbol{r}, t)$, one uses the least action principle to variationally derive the timedependent Schrödinger equation, see e.g., [12],

$$
\begin{aligned}
& i \hbar \partial \Psi(\boldsymbol{r}, t) / \partial t=\mathrm{H}(\boldsymbol{r}, t) \Psi(\boldsymbol{r}, t) \text { or } \\
& {[i \hbar \partial / \partial t-\mathrm{H}(\boldsymbol{r})] \Psi(\boldsymbol{r}, t) \equiv \mathrm{A}(\boldsymbol{r}, t) \Psi(\boldsymbol{r}, t)=0 .}
\end{aligned}
$$

This physical quantity is represented by the quantum-mechanical action integral in state $\Psi$,

$$
\mathcal{A}[\Psi]=\int\langle\Psi(t)|\mathrm{A}(t)| \Psi(t)\rangle d t \equiv \int A[\Psi(t)] d t
$$

defined by the action operator $\mathrm{A}(\boldsymbol{r}, t)$ of Eq. (64). Indeed the associated Euler equation of the vanishing functional derivative

$$
\delta \mathcal{A}[\Psi] / \delta \Psi^{*}(\boldsymbol{r}, t)=\mathrm{A}(\boldsymbol{r}, t) \Psi(\boldsymbol{r}, t)=0,
$$

which marks the stationary property of $\mathcal{A}[\Psi]$, is then seen to directly recover Eq. (64).

We therefore conclude, that the phase/current part of the information measure in quantum mechanics is indeed essential for distinguishing the amount of information of states exhibiting the same modulus (probability) part and differing in their phases (currents). The variational principles involving the generalized Fisher measure have been examined elsewhere $[3,9]$. In the next section we examine a general EPI rule involving the quantum Shannon entropy.

\section{Maximum entropy principles and "thermodynamic" equilibrium states}

Following Eq. (53) one might expect that a general maximum-entropy rule for determining the molecular equilibrium should involve both the thermodynamic (physical) constraint $E[\psi]=E_{0}$ and the geometrical requirement of the unit norm of the optimum wave function:

$$
\max _{\psi}\left\{S[p, \Phi]-\lambda\left(E[\psi]-E_{0}\right)-\mu(\langle\psi \mid \psi\rangle-1)\right\}
$$


In this complex variational problem the two conjugate states involved, $\psi$ and $\psi^{*}$, determine the two independent variational degrees of freedom which uniquely identify the real and imaginary components of the wave-function. In what follows we examine the effect of general variations $\psi^{*} \rightarrow \psi^{*}+\delta \psi^{*}$ on the auxiliary entropy functional of Eq. (67).

We first observe that the functional derivative of the quantum-mechanical entropy $S[p, \Phi]$ of Eq. (46) with respect to $\psi^{*}$ reads:

$$
\begin{aligned}
\frac{\delta S[p, \Phi]}{\delta \psi^{*}(\boldsymbol{r})} & =\left\{\frac{\partial S^{\text {clas. }}[p, \Phi]}{\partial p(\boldsymbol{r})}+\frac{\partial S^{\text {nclas. }}[p, \Phi]}{\partial p(\boldsymbol{r})}\right\} \frac{\partial p(\boldsymbol{r})}{\partial \psi^{*}(\boldsymbol{r})}+\frac{\partial S^{\text {nclas. }}[p, \Phi]}{\partial \Phi(\boldsymbol{r})} \frac{\partial \Phi(\boldsymbol{r})}{\partial \psi^{*}(\boldsymbol{r})} \\
& =\{[2 \Phi(\boldsymbol{r})-\ln p(\boldsymbol{r})]+(i-1)\} \psi(\boldsymbol{r}) .
\end{aligned}
$$

Hence, the functional derivative with respect to $\psi^{*}$ of Eq. (67) generates the following Euler equation determining the optimum $\psi(\boldsymbol{r})=\psi^{0}(\boldsymbol{r})=\mathrm{R}^{0}(\boldsymbol{r}) \exp \left[i \Phi^{0}(\boldsymbol{r})\right]$, i.e., $p(\boldsymbol{r})=p^{0}(\boldsymbol{r})=\left[R^{0}(\boldsymbol{r})\right]^{2}$ :

$$
\left\{\left[2 \Phi^{0}(\boldsymbol{r})-\ln p^{0}(\boldsymbol{r})\right]-\lambda \mathrm{H}(\boldsymbol{r})+(i-1-\mu)\right] \psi^{0}(\boldsymbol{r})=0 .
$$

Thus, after dividing by $\lambda \neq 0$ (marking an essential energy constraint) it formally recovers the eigenvalue problem of the Hamiltonian [Eq. (49)] for

$$
\begin{aligned}
2 \Phi^{0}(\boldsymbol{r}) & =\ln p^{0}(\boldsymbol{r}) \quad \text { or } \quad \Phi^{0}(\boldsymbol{r})=\ln \left[p^{0}(\boldsymbol{r})\right]^{1 / 2}=\ln R^{0}(\boldsymbol{r}), \mu=-1 \quad \text { and } \\
\lambda & =i / E_{0} .
\end{aligned}
$$

It could be directly verified using Eq. (59) that this optimum state

$$
\psi^{0}(\boldsymbol{r})=R^{0}(\boldsymbol{r}) \exp \left[i \Phi^{0}(\boldsymbol{r})\right]=R^{0}(\boldsymbol{r}) \exp \left[i \ln R^{0}(\boldsymbol{r})\right]=R^{0}(\boldsymbol{r})(\cos 1+i \sin 1)^{\ln R^{0}(\boldsymbol{r})},
$$

indeed reproduces the electronic energy expression of Eq. (57):

$$
E\left[\psi^{0}\right]=\int R^{0}(\boldsymbol{r})^{2} v(\boldsymbol{r}) d \boldsymbol{r}+\frac{\hbar^{2}}{2 m} \int\left[\nabla R^{0}(\boldsymbol{r})\right]^{2} d \boldsymbol{r} .
$$

Therefore, since $E\left[\psi^{0}\right]=E\left[\psi_{0}\right]$ or $E\left[\psi^{0}\right]=E_{v}\left[p_{0}\right]$, this nondegenerate ground state uniquely identifies $R^{0}(\boldsymbol{r})=R_{0}(\boldsymbol{r})$ and $p^{0}(\boldsymbol{r})=p_{0}(\boldsymbol{r})$, in accordance with the familiar Hohenberg-Kohn theorems [13] of the modern Density Functional Theory (DFT). However, since its phase is position-dependent this optimum ("thermodynamic") state also exhibits the nonvanishing probability current [see Eq. (7)]:

$$
\boldsymbol{j}^{0}(\boldsymbol{r})=(\hbar / m) p_{0}(\boldsymbol{r}) \nabla \Phi^{0}(\boldsymbol{r})=(\hbar / m) R_{0}(\boldsymbol{r}) \nabla R_{0}(\boldsymbol{r}) .
$$

Thus, the lowest maximum-entropy ("thermodynamic") equilibrium state $\psi^{0}$ differs from the true eigenstate $\psi_{0}=R_{0}$ of the Hamiltonian [Eq. (56)], for which the probability current identically vanishes, although they both exhibit the same energy. 
In the time-dependent maximum entropy rule for determining the optimum nonstationary state $\Psi(\boldsymbol{r}, t)=\mathrm{R}(\boldsymbol{r}, t) \exp [i \Phi(\boldsymbol{r}, t)]$ and the particle distribution $p(\boldsymbol{r}, t)=$ $\mathrm{R}(\boldsymbol{r}, t)^{2}$, defined by the real modulus factor $R(\boldsymbol{r}, t)$ and phase function $\Phi(\boldsymbol{r}, t)$, the stationary-action constraint $\mathcal{A}[\Psi]=\mathcal{A}^{0}$ replaces the fixed-energy requirement of the stationary problem:

$$
\max _{\Psi}\left\{S[p, \Phi]-\lambda\left(\mathcal{A}[\Psi]-\mathcal{A}^{0}\right)-\mu(\langle\Psi \mid \Psi\rangle-1)\right\}
$$

The relevant Euler equation, from the functional differentiation of the preceding equation with respect to $\Psi^{*}$, now reads:

$$
\left.\left\{\left[2 \Phi^{0}(\boldsymbol{r}, t)-\ln p^{0}(\boldsymbol{r}, t)\right]-\lambda \mathrm{A}(\boldsymbol{r}, t)+(i-1-\mu)\right]\right\} \Psi(\boldsymbol{r}, t)=0 .
$$

For $\lambda \neq 0$ it reproduces the time-dependent SE of Eq. (64), $\mathrm{A}(\boldsymbol{r}, t) \Psi(\boldsymbol{r}, t)=0$, for

$$
\begin{aligned}
2 \Phi^{0}(\boldsymbol{r}, t) & =\ln p^{0}(\boldsymbol{r}, t) \quad \text { or } \quad \Phi^{0}(\boldsymbol{r}, t)=\ln \left[p^{0}(\boldsymbol{r}, t)\right]^{1 / 2}=\ln R^{0}(\boldsymbol{r}, t), \text { and } \\
\mu & =i-1 .
\end{aligned}
$$

Hence, the optimum time-dependent "thermodynamic" state reads:

$$
\Psi^{0}(\boldsymbol{r}, t)=R^{0}(\boldsymbol{r}, t) \exp \left[i \ln R^{0}(\boldsymbol{r}, t)\right]
$$

Its probability distribution $p^{0}(\boldsymbol{r}, t)=\mathrm{R}^{0}(\boldsymbol{r}, t)^{2}$ and current

$$
j^{0}(\boldsymbol{r}, t)=(\hbar / m) p_{0}(\boldsymbol{r}, t) \nabla \Phi^{0}(\boldsymbol{r}, t)=(\hbar / m) R^{0}(\boldsymbol{r}, t) \nabla R^{0}(\boldsymbol{r}, t)
$$

satisfy the probability continuity equation (17), which is a direct consequence of SE. Moreover, the corresponding phase density and current (see Sect. 3) satisfy the local balance of Eq. (24).

\section{Conclusion}

The quantum generalizations of the classical Fisher and Shannon information measures, functionals of the particle probability distribution, have been introduced and discussed. They are applicable to the complex probability amplitudes (wave functions) of the quantum mechanical description. Their associated continuity equations have been examined and the associated nonclassical information sources, linked to the wave function phase or probability current densities, have been identified. In particular the phase-current density have been introduced, which complements the familiar probability-current concept.

The maximum quantum-entropy principle generates the equilibrium ("thermodynamic") states in which the phase component is directly linked to modulus part of the system wave function:

$$
\psi^{0}(\boldsymbol{r})=R_{0}(\boldsymbol{r}) \exp \left[i \ln R_{0}(\boldsymbol{r})\right] \quad \text { or } \quad \Psi^{0}(\boldsymbol{r}, t)=R^{0}(\boldsymbol{r}, t) \exp \left[i \ln R^{0}(\boldsymbol{r}, t)\right] .
$$


Thus the lowest equilibrium state $\psi^{0}$ derived from the general maximum-entropy rule differs from the true ground-state $\psi_{0}$ (lowest eigenstate of the Hamiltonian), both corresponding to the same energy $E_{0}$ and probability distribution $p_{0}$, by exhibiting nonvanishing probability current $j^{0}$. Its presence effectively increases the entropy (electron indeterminacy) content above the classical level determined by the particle probability alone. Therefore, the amount of information received by removing this indeterminacy, as a result of the particle localization experiment, is larger in $\psi^{0}$ than in $\psi_{0}$.

It should be observed, however, that the $p_{0}$-constrained entropy principle gives predictions fully consistent with the ordinary quantum mechanics. Indeed, by the Hohenberg-Kohn theorem of DFT the fixed ground-state density $\rho_{0}=N p_{0}$ then uniquely determines the system energy $E_{v}\left[\rho_{0}\right]=E_{v}\left[p_{0}\right] \equiv E_{0}$, so that the energy constraint is redundant in the general entropy principle of Eq. (67). Therefore, optimization of $\psi$ then amounts to a search for the optimum phase $\Phi$ :

$$
\max _{\Phi}\left\{S\left[p_{0}, \Phi\right]-\mu\langle\psi \mid \psi\rangle\right\}
$$

It gives rise to the associated Euler equation $(i-\mu) \psi=0$, which is automatically satisfied for $\mu=i$, leaving $\Phi$ completely undetermined.

To summarize, the general entropy principle, which allows variations in probability distribution and hence also the system energy, predicts the stationary thermodynamic state of Eq. (79), which differs from the true ground state of the molecular system in question, while its density/energy-contrained principle of Eq. (80) does not resolve the familiar arbitrariness of the phase in quantum mechanics. As also argued in Section 4, the quantum-generalized gradient measure of the Fisher-type determines the ground state uniquely, predicting the space-independence of the resulting phase. Indeed, since $p_{0}$ fixes the classical part of the information content, the minimum of the quantum Fisher information is determined by the lowest value of its nonclassical part of Eq. (34), i.e., the vanishing probability current density: $\boldsymbol{j}=\boldsymbol{0}$. This further implies [see Eq. (7)] the space-independent phase of the ground-state wave function [see Eq. (30)]. It would thus seem that this state of the vanishing current everywhere represent the state of the absolute maximum uncertainty in electronic state. Indeed, any presence of a finite current density increases the information (structure, "order") in the ground-state of the system electrons, thus decreasing a measure of their complementary entropy (uncertainty, "disorder") descriptor.

Open Access This article is distributed under the terms of the Creative Commons Attribution License which permits any use, distribution, and reproduction in any medium, provided the original author(s) and the source are credited.

\section{References}

1. R.A. Fisher, Proc. Cambridge Phil. Soc. 22, 700 (1925)

2. C.E. Shannon, Bell System Tech. J. 27, 379, 623 (1948)

3. R.F. Nalewajski, Int. J. Quantum Chem. 108, 2230 (2008)

4. R.F. Nalewajski, Foundations of chemistry, in Quantum Theory of Atoms and Molecules, ed. by C. Matta (in press) 
5. B.R. Frieden, Physics from the Fisher Information-A Unification, 2nd edn. (Cambridge University Press, Cambridge, 2004)

6. R.F. Nalewajski, J. Math. Chem. (2012). doi:10.1007/s10910-012-0054-2

7. R.F. Nalewajski, Information Theory of Molecular Systems (Elsevier, Amsterdam, 2006)

8. R.F. Nalewajski, Information Origins of the Chemical Bond (Nova, New York, 2010)

9. R.F. Nalewajski, Perspectives in Electronic Structure Theory (Springer, Berlin, 2012)

10. C.F. von Weizsäcker, Z. Phys. 96, 431 (1935)

11. H.B. Callen, Thermodynamics: An Introduction to the Physical Theories of the Equilibrium Thermostatics and Irreversible Thermodynamics (Wiley, New York, 1960)

12. E.K.U. Gross, J.F. Dobson, M. Petersilka, in, Topics in Current Chemistry, vol. 181: Density Functional Theory II, ed. by R.F. Nalewajski (Springer, Heidelberg, 1996), p. 81

13. P. Hohenberg, W. Kohn, Phys. Rev. 136, 864 (1964) 